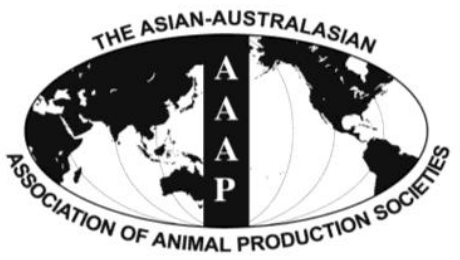

Asian Australas. J. Anim. Sci.

Vol. 26, No. 11 : 1644-1650 November 2013

http://dx.doi.org/10.5713/ajas.2013.13157

Www.ajas.info

pISSN 1011-2367 elSSN 1976-5517

\title{
Molecular Characterization and Expression Analysis of Ribosomal Protein S6 Gene in the Cashmere Goat (Capra hircus)
}

\author{
Bao Wenlei ${ }^{\mathrm{a}}$, Hao Xiyan ${ }^{\mathrm{a}}$, Zheng Xu, Liang Yan, Chen Yuhao, Wang Yanfeng, and Wang Zhigang* \\ College of Life Science, Inner Mongolia University, Hohhot 010021, China
}

\begin{abstract}
Ribosomal protein (rp) S6 is the substrate of ribosomal protein S6K (S6 kinase) and is involved in protein synthesis by mTOR/S6K/S6 signaling pathway. Some S6 cDNA have been cloned in mammals in recent years but has not been identified in the goat. To facilitate such studies, we cloned the cDNA encoding Cashmere goat (Capra hircus) S6 (GenBank accession GU131122) and then detected mRNA expression in seven tissues by real time PCR and protein expression in testis tissue by immunohistochemisty. Sequence analysis indicated that the obtained goat S6 was a 808 bp product, including a 3 ' untranslated region of 58 bp and an open reading frame of $750 \mathrm{bp}$ which predicted a protein of 249 amino acids. The predicted amino acid sequence was highly homologous to cattle, human, mouse and rat S6. Expression analysis indicated S6 mRNA was expressed extensively in detected tissues and S6 protein was expressed in testis tissue. (Key Words: Cashmere Goat, Ribosomal Protein (S6) Gene, Expression Pattern, The Mammalian Target of Rapamycin (mTOR))
\end{abstract}

\section{INTRODUCTION}

The ribosomal protein S6 (rpS6, S6) is a critical component of the $40 \mathrm{~S}$ ribosomal subunit and the major phosphoprotein of the ribosome. S6 is multiple phosphorylated on serine residues in various cells by the stimulation of different agents such as mitogens, viruses, oncogene products, phorbol esters, progesterone and insulin.

Some reports suggested that S6 may affect protein synthesis. The phosphorylation of $\mathrm{S} 6$ is thought to be required for the translation of a subset of mRNAs that contain a $5^{\prime}$-oligopyrimidine tract at their transcriptional start sites (5'-terminal oligopyrimidine, 5'-TOP) (Iwenofu et al., 2008; Jefferies et al., 1997; Klann et al., 2004). 5'-TOP mRNAs may number as few as 100-200, but they can account for 20 to $30 \%$ of total cellular mRNA. They encode many components of the translational apparatus, including ribosomal proteins and elongation factors that are necessary for cell cycle progression, growth and proliferation (Boylan et al., 2001; Ruvinsky and Meyuhas, 2006; Ruvinsky et al., 2009). In recent years, a growing number of S6 studies have focused on new functions and relationships with other

\footnotetext{
* Corresponding Author: Wang Zhigang. Tel: +86-471-4995867 (8008), Fax: +86-471-4992435, E-mail: 1swzg@imu.edu.cn

${ }^{\text {a }}$ These authors contributed equally to this work.

Submitted Mar. 17, 2013; Accepted May 13, 2013; Revised Jun. 27, 2013
}

molecules. Casein kinase 1 (CK1)-dependent phosphorylation of rpS6 promotes its association with the mRNA capbinding complex in vitro and protein phosphatase 1 (PP1) antagonizes rpS6 $\mathrm{C}$ terminus phosphorylation and cap binding in intact cells (Hutchinson et al., 2011; Li et al., 2012). Rabin8, a guanine nucleotide exchange factor for Rab8 GTPase, suppresses phosphorylation of Ser235/Ser236 in ribosomal protein S6 (Parkhitko et al., 2011). Phosphorylated S6 has a different cytoplasmic/nuclear distribution (Rosner et al., 2011) and a well-established nuclear function beyond its role in ribosome (Chen and Dittmer, 2011). These findings further our understanding of S6 phospho-regulation and suggest the existence of other important roles in cytophysiology.

S6 was the first identified member of the p70 ribosomal S6 kinase (S6K) substrates family, and its phosphorylation has been studied thoroughly at the structural, kinetic and functional level. The phosphorylation sites in S6 have been mapped to five clustered residues, Ser235, Ser236, Ser240, Ser244, and Ser247, whose location at the C terminus of higher eukaryotes is evolutionarily conserved (Krieg et al., 1988; Ruvinsky et al., 2009). It has been proposed that phosphorylation progresses in an ordered manner and that Ser236 is the primary phosphorylation site (Flotow and Thomas, 1992; Ruvinsky and Meyuhas, 2006).

$\mathrm{S} 6 \mathrm{~K}$ is an important downstream effector of the 
mammalian target of rapamycin (mTOR) and mTOR/S6K/S6 signaling pathway is regulated in response to cytokines, nutrients and growth factors. Studies over the past decade have demonstrated that mTOR/S6K/S6 axis is involved in protein synthesis, cell growth and apoptosis, embryo development, and cancer (Laplante and Sabatini, 2012); (Magnuson et al., 2012); (Fenton and Gout, 2011). Meanwhile, the mTOR signaling pathway is involved in transcription, autophage and metabolism disease (Schmelzle and Hall, 2000; Proud, 2002; Wullschleger et al., 2006). The mTOR/S6K/S6 axis has emerged as a major effector of cell growth and proliferation by regulation of protein synthesis.

The S6 cDNA and S6 have been investigated extensively in human, mouse, rat and bovine, whereas it has not been studied yet in goat due to the lack of basic data about the gene, gene expression and function. To facilitate the further study of the function and regulation of S6 in Inner Mongolia Cashmere goat cells, we cloned full-length S6 gene cDNA, measured its gene products in various tissues by quantitative real-time PCR and immunohistochemistry.

\section{MATERIALS AND METHODS}

Inner Mongolia Cashmere goats were bred on a natural diet in Inner Mongolia, China. Tissues including brain, heart, testis, liver, spleen, kidney and lung were collected from five male adult goats after slaughter in a commercial goat slaughter farm in the spring. Tissue samples were flash frozen in liquid nitrogen immediately after harvesting and stored at $-80^{\circ} \mathrm{C}$.

\section{Cell cultures}

Inner Mongolia Cashmere goat fetal fibroblasts $(\mathrm{GFb}$ cells) were cultured in DMEM/F12 (Gibco) supplemented with $10 \%$ fetal bovine serum (FBS, HyClone Laboratories, Inc. Logan, UT USA) and $100 \mathrm{U} / \mathrm{mL}$ penicillin G, 100 $\mathrm{mg} / \mathrm{mL}$ streptomycin (Sigma-Aldrich, Inc. St. Louis USA), and maintained in a monolayer culture at $37^{\circ} \mathrm{C}$ in humidified air with $5 \% \mathrm{CO}_{2}$. Cellular morphology was observed through a light microscope during culture and experiments.

\section{RNA extraction and cDNA synthesis}

Total RNA was prepared by RNAzol (RNAiso Plus, TaKaRa Co. Ltd., China) from brain, heart, testis, liver, spleen, kidney, lung and fetal fibroblasts of Inner Mongolia Cashmere goat. RNAs were reversely transcribed with an oligo(dT) $)_{12-18}$ primer using an AMV 1st strand cDNA (cDNA1) Synthesis kit (Takara Co. Ltd., China) according to the instructions of the manufacturer. An input of $1 \mu \mathrm{g}$ total RNA was used for each reaction. DNA was removed by DNase I (RNase-free).

\section{Cloning and sequencing of the gene encoding Cashmere goat $\mathrm{S6}$ protein}

The S6 cDNA fragment was amplified by PCR which was carried out for 35 cycles with cDNA1 as template at the appropriate annealing temperature $\left(50.5^{\circ} \mathrm{C}\right)$ for the following pairs of primers, forward: $5^{\prime}$ - ATGAAGCT GAAYATCTCYTTCC-3', reverse: 5' -TCATTTTTGAC TGGACYCAGAYT - $3^{\prime}$ ( $\mathrm{Y}$ means $\mathrm{C}$ or $\mathrm{T}$ ). The primers were designed by comparing the nucleotide sequences of S6 genes released in GenBank. The predicted fragment length was $750 \mathrm{bp}$. The PCR products were then electrophoresed and photos were taken by an electronic UV transilluminator (UVItec, UK). The PCR products were cloned into plasmid and sequenced by an ABI PRISM 377XL DNA Sequencer (Applied Biosystems, Inc. Foster City, CA).

\section{$3^{\prime}$ rapid amplification of cDNA ends ( $3^{\prime}$ RACE)}

RNAs were reversely transcribed with $3^{\prime}$ Rapid Amplification of cDNA Ends (3' RACE) 1st strand cDNA (cDNA2) Synthesis kit and a 3' RACE Adaptor primer was used according to the instructions of the manufacturer (Takara Co. Ltd., China). The amplification of 3' UTR fragment was carried out with cDNA2 as template for 35 cycles at the appropriate annealing temperature $\left(50.5^{\circ} \mathrm{C}\right)$ for the following pairs of primers, forward: 5' - GAAAGCC CCTAAAC AAAGACGGT -3', reverse: 5' - TACCGTCG TTCCACTAGTGATTT-3'. 3' RACE generated a single fragment of $356 \mathrm{bp}$.

\section{Quantitative real-time PCR analysis of S6 mRNA distribution in different tissues}

Tissue distribution of S6 mRNA was performed using quantitative real-time PCR analysis. The amplification of S6 cDNA fragment was carried out with the cDNA1 as template by Real-time PCR using the following pairs of primers, forward: 5' - TTGTGGATGCCAATC TGAGTGTT -3', reverse: 5' -GGTTTCTTACCGTCTTTGTTTAGGG $3^{\prime} ; \beta$-actin fragment was amplified with the pairs of primers, forward: 5' - TGGCACCACACCTTCTACA ACGAGC -3', reverse: 5' -CGTCCCCAGAGTCCATGACAATG -3'. The quantitative real time PCRs were performed with a Bio-rad Chromo 4 PCR System using SYBR® Premix Ex TaqTM (Perfect Real Time) (TaKaRa Co. Ltd., China). Amplification was conducted with an initial denature action step at $95^{\circ} \mathrm{C}$ for $5 \mathrm{~min}$, followed by $95^{\circ} \mathrm{C}$ for $5 \mathrm{~s}, 60^{\circ} \mathrm{C}$ for $30 \mathrm{~s}$, and $72^{\circ} \mathrm{C}$ for $40 \mathrm{~s}, 40$ cycles, $72^{\circ} \mathrm{C}$ for $10 \mathrm{~min}$ and a final melting curve. Three technical replicas were performed. Delta CT $(\Delta \mathrm{CT})$ values were calculated and tissue distribution in the test genes expression was finally calculated. A statistical evaluation of real-time PCR results 
was executed using one-way analysis of variance (ANOVA) to compare test gene expression in different tissues.

\section{Immunohistochemistry}

The tissue samples were fixed in $4 \%$ paraformaldehyde, an alcohol gradient- dehydrated and infiltrating waxincubated, then paraffin-embedded and sectioned. Sections were deparaffinised in an alcohol gradient, blocked with normal nonimmune animal serum, incubated with anti-S6 antibody (Cell Signaling Technology, Inc. Danvers, MA USA; $1: 100)$ at $4^{\circ} \mathrm{C}$ overnight. Subsequently, sections were incubated with biotinylated anti-rabbit $\operatorname{IgG}$ antibody (1:50) and stained with DAB (3, 3'-diaminobenzidine tetrahydrochloride) using UltraSebsitive TM SP kit (Maixin_Bio, Fuzhou, China) according to the manufacturer's instructions. Before mounting, sections were counter-stained with haematoxylin. As negative controls, sections were immunostained in the absence of primary antibodies for each tissue and incubated in PBS.

\section{Bioinformatics analysis}

Nucleotide sequence of goat S6 cDNA and deduced amino acid sequence were identified by the NCBI BLAST program (http://www.ncbi.nlm.nih.gov/BLAST/). Open reading frames (ORFs) and theoretical molecular weight of deduced polypeptides were predicted by the Protein property calculator (http://www.basic.northwestern.edu/ biotools/proteincalc.html). The protein Isoelectric Point was predicted by the calculation of protein isoelectric point http://isoelectric.ovh.org/. Protein domain analysis was identified by the SMART program (http://smart.emblheidelberg.de/). Protein prosite patterns analysis were identified by the Psite program (http://www.softberry.com). The model of certain regions was generated using SWISSMODEL Workspace (Arnold et al., 2006; Guex and Peitsch, 1997; Schwede et al., 2003).

\section{Statistical analysis}

Descriptive statistics were generated for all quantitative data with presentation of mean \pm SD. For each assay, triplicate parallel observations were examined.

\section{RESULTS}

\section{cDNA cloning and sequence analysis}

The S6 cDNA (GenBank accession GU131122) from Inner Mongolia Cashmere goat includes an open reading frame of $750 \mathrm{bp}$ and a $58 \mathrm{bp}$ - residue fragment of the $3^{\prime}$ untranslated region containing a polyadenylation signal locating in nucleotide 769 774(AATAAA), 22 bp upstream of the poly(A) tract. The full cDNA sequence has revealed a $96 \%$ identity to bovine and $90 \%, 87 \%$ and $87 \%$ identity to human, mouse and rat, respectively. The amino acid sequence is $100 \%$ identical to the bovine and $99 \%$ to the human, mouse and rat. The 3' UTR sequence of S6 is quite short, only $18 \mathrm{bp}$. Here, the $3^{\prime}$ UTR sequence is defined as the mRNA region spanning from the stop codon (excluded) to the poly-adenylation signal site (excluded). We compared its length of $3^{\prime}$ UTR with those of five goat gene cDNAs with intact $3^{\prime}$ UTR released in the GenBank, mTOR, S6K1, $G P x 4, K 26$ and $L D H-A$, the $3^{\prime}$ UTR of S6 mRNA is much shorter than those five nonribosomal protein genes (Figure $1)$.

\section{Primary and secondary structure of the Cashmere goat S6 protein}

The activity sites within S6 protein were predicted by the Psite software and the results showed that there are three $\mathrm{N}$-glycosylation sites, three cAMP- and cGMP-dependent protein kinase phosphorylation sites, six Protein kinase $\mathrm{C}$ phosphorylation sites (Figure 2A).

The deduced primary structure of the Cashmere goat

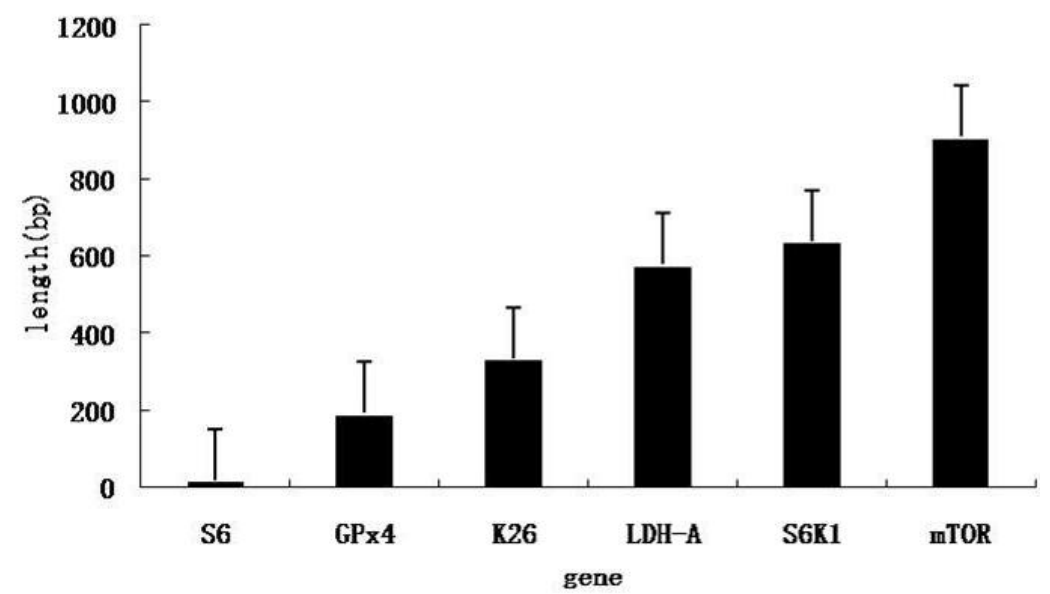

Figure 1. Size distribution of the 3' UTRs of six available data. The 3' UTR length expressed as number of nucleotides between the stop codon and the polyadenylation signal (AATAAA). mTOR (HM114224), S6K1 (GU144017), S6 (GU131122), GPx4 (GU131344), K26 (GU084153.1) and LDH-A (GU144018). 


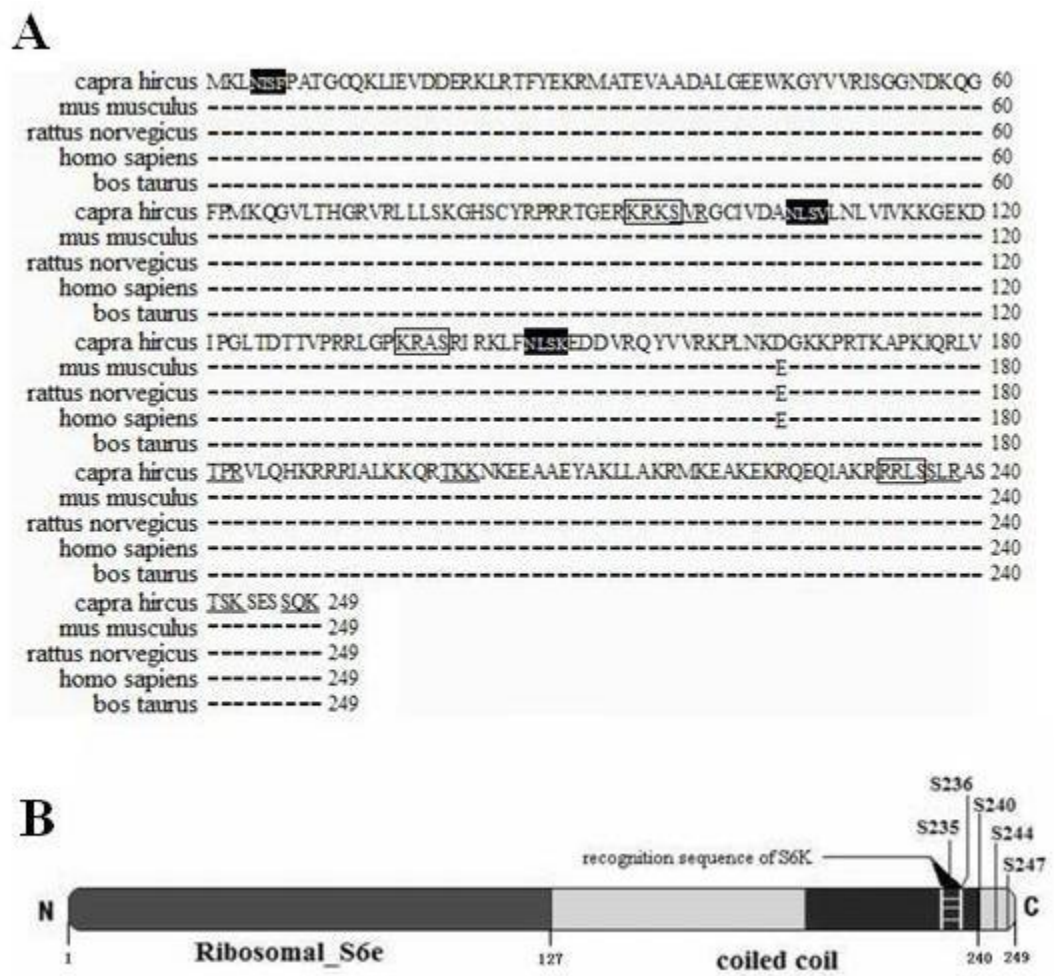

Figure 2. Deduced amino acid sequence of ribosomal protein S6. (A) Alignment of the amino acid sequences of capra hircus (GU131122), mus musculus (NP-033122.1), rattus norvegicus (NP-058856.1), homo sapiens (NP-001001.2), bos taurus (NP-001015548.1). Predicted N-glycolsylation sites (Asn-Xaa-Ser/Thr) are marked by black shading. Protein kinase C phosphorylation sites (Ser/Thr-XaaLys/Arg) are underlined, cAMP- and cGMP-dependent protein kinase phosphorylation sites (Arg/Lys-Xaa-Ser/Thr) are marked by pane, and all these were determined using the Psite software. (B) There are two main domains in the rpS6 which is 249 amino acids in length. The ribosomal_S6e domain is the criterion to classify eukaryotic ribosomal proteins. A coiled coil is a structural motif in proteins, in which 2-7 alpha-helices are coiled together like the strands of a rope. The domains of S6 were predicted with SMART Software. The phosphorylation sites in rpS6 have been mapped to five clustered residues, Ser235, Ser236, Ser240, Ser244 and Ser247, whose location at the $\mathrm{C}$ terminus of higher eukaryotes is evolutionarily conserved. The amino acids in 231-236 are the recognition sequence of S6K, whose sequence context is RRRLSS.

ribosomal protein S6 consists of 249 amino acids, and the predicted molecular weight is $M_{\mathrm{r}} 28,785 \mathrm{Da}$ for the unmodified protein, the estimated isoelectric point $(\mathrm{p} I)$ is 11.13. The basic amino acids comprise $14.5 \%$ lysine, $12.9 \%$ arginine, $9.2 \%$ leucine and $6.8 \%$ valine of the total. There is a ribosomal_S6e domain (residues 1 to 127) and a coiled coil (residues 94 to 239). The fragment of residues (231 to 236 ) is the recognition sequence of S6K (Figure 2B). There is a dominating region in goat ribosomal protein S6 protein, as calculated by the SWISS-MODEL workspace - a Ribosomal_S6e domain from 1 to 127, the quaternary structure is shown in Figure 3.

\section{Tissue distribution of Cashmere goat S6 mRNA and S6 protein}

The relative abundance of S6 mRNA was assessed in
A

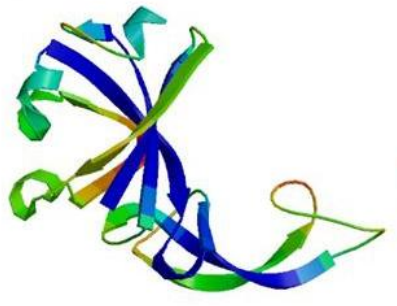

B

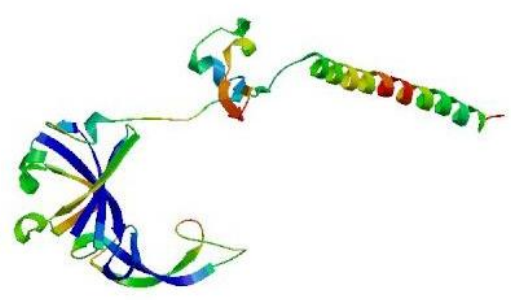

Figure 3. Three-dimensional model of S6 regions. (A) The model of the N-terminal region, a Ribosomal_S6e domain, residues 1 to 127. (B) The model of goat S6 main region, residues 1 to 219. 


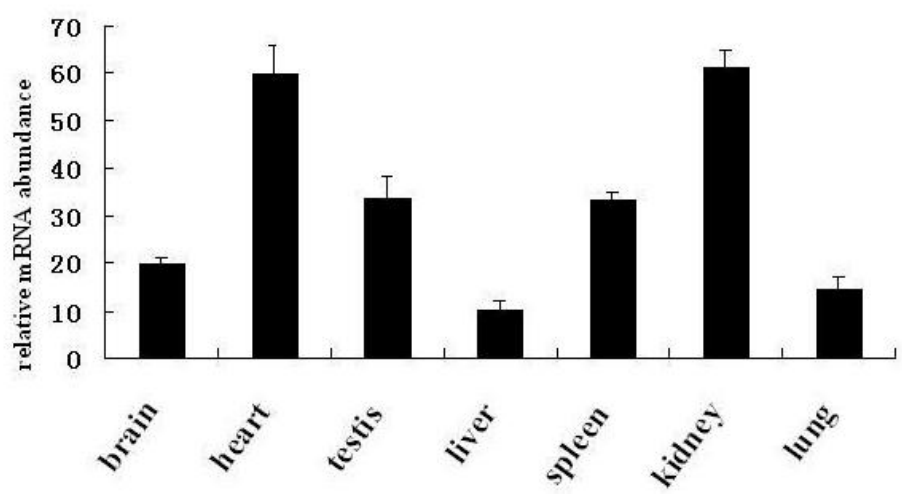

Figure 4. Tissue distribution of S6 transcripts. S6 transcripts were analyzed by quantitative real-time RT-PCR with SYBR Premix Ex Taq (Perfect Real Time) system. The mRNA level is higher in heart and kidney compared with brain, liver, spleen, lung and testis.

brain, heart, testis, liver, spleen, kidney and lung tissues from Cashmere goats by quantitative real-time PCR analyses. The results indicated that the mRNA level is higher in heart and kidney compared with brain, liver, spleen, lung and testis (Figure 4). Then the protein expression of S6 was detected in testis of Inner Mongolia cashmere goats by immunohistochemistry technique. As shown in Figure 5, the tissue cells are brown in the experiment group (Figure 5B) which was incubated with anti- ribosomal protein $\mathrm{S} 6$ protein antibody, while it is colorless in control (Figure 5A) which was incubated with PBS, suggesting that the S6 protein is expressed in testis tissue.

\section{DISCUSSION}

Ribosomal protein (rp) S6 is a major substrate of protein kinases in eukaryotic ribosomes. In all living organisms, the ribosome is a vital organelle which is responsible for protein synthesis. Production of mature ribosomes consisting of rRNAs and ribosomal proteins requires a highly coordinated multistep process. The ORF of cloned goat $S 6$ cDNA encodes the same amino acid sequence as the bovine and the deduced amino acid sequence has revealed a 99\% identity to those of the human, mouse and rat.

The predicted S6e domain locates in the N-terminal of goat S6 protein and the ribosomal protein S6e family was reported to reside in evolutionarily highly conserved peptide clusters (Kundu-Michalik et al., 2008). This implies that $S 6$ genes are highly conserved which is consistent with its important function of protein synthesis.

In the present study, 3' UTR of the goat $S 6$ gene is quite short, only $18 \mathrm{bp}$. In fact, this feature is not only found in goat, it was reported that the $3^{\prime}$ UTR of all ribosomal protein mRNAs is much shorter than most cellular mRNAs (Ledda et al., 2005). It is known that cis-acting control elements affecting mRNA stability often locate in the $3^{\prime}$ UTR. The effect of 3' UTR length on mRNA's translation efficiency has been studied in transiently transfected Chinese hamster ovary (CHO) cells using firefly luciferase (luc) reporter gene and the data indicated that $3^{\prime}$ UTR length plays an important role in determining both the translational efficiency and the stability of an mRNA (Tanguay and Gallie, 1996). The finding that all rp-protein mRNAs (rp-mRNAs) are characterized by a very short $3^{\prime}$ UTR suggests that this structural feature might be relevant for the translational regulation function of this mRNAs. There are several possibilities regarding the $3^{\prime}$ UTR length and its potential functions. The first is that the $3^{\prime}$ UTR length has an effect on the translation in resting cells but not in stimulated cells (Ledda et al., 2005). The second is that S6 mRNAs could exist a longer time as templates for translating, because the short 3' UTR of S6 has fewer sites

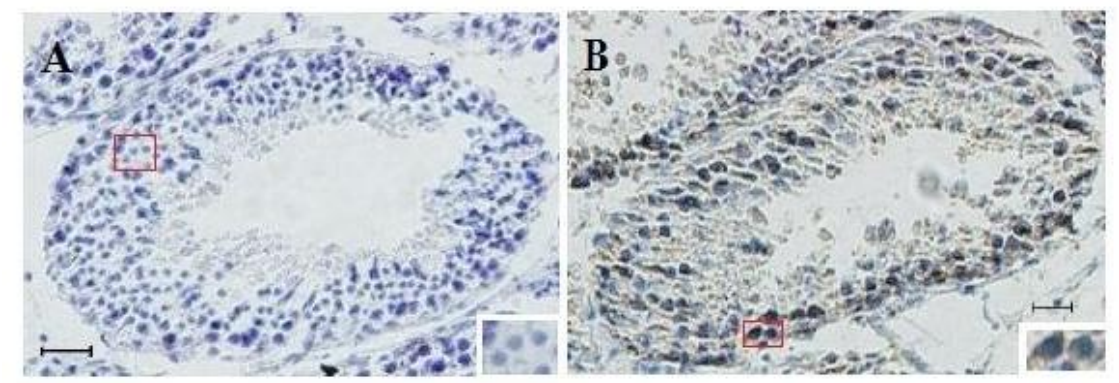

Figure 5. The expression of S6 protein in testis tissue. A section was incubated in PBS instead of primary antibody. B section was incubated with anti-S6 antibody (1:100). S6 was expressed in the brown tissue cells. Magnification $=200 \times$ and $400 \times$, scale bars $25 \mu \mathrm{m}$. 
for miRNA regulation through RNA interference (RNAi) (Stark et al., 2005; Bartel, 2009; Zhang and Su, 2009). The third is that the short $3^{\prime}$ UTR of rp-mRNAs might correlate with its $5^{\prime}$ UTR structural feature. It has been demonstrated that $5^{\prime}$-terminal oligopyrimidine $\left(5^{\prime}\right.$ TOP) is another structural feature of rp-mRNAs. The $5^{\prime}$ UTR of rp-mRNAs, containing the typical $5^{\prime}$ TOP sequence, is the cis-acting element responsible for the regulation (Iwenofu et al., 2008). We don't know how they work together, but the closed loop model (Kahvejian et al., 2001; Mazumder et al., 2003; Svitkin and Sonenberg, 2004) appears to be the better explanation. In this model, the connection between the $3^{\prime}$ and the $5^{\prime}$ - ends of the mRNA play a role in selecting intact mRNAs for translation and protecting mRNA from degradation.

There is a great amount of evidence showing that S6 is a downstream molecule of mTOR (Hay and Sonenberg, 2004). It is well known that rapamycin represses $5^{\prime}$ TOP mRNA translation. Previously, the function of S6K was also thought to correlate with this effect (Flotow and Thomas, 1992). However, recent studies with the knockout of both forms of S6K disapprove this hypothesis (Pende et al., 2004). So how does mTOR regulate $5^{\prime}$ TOP mRNA translation? It is understandable that mTOR can regulate the activity of TIF-IA, an essential initiation factor that is associated with the initiation-competent form of RNA polymerase I (Pol I), through PP2A (protein phosphatase 2A) (Peterson et al., 1999), a downstream effector of mTOR (Mayer et al., 2004). So rapamycin could inhibit transcription of rRNA by Pol I. mTOR coordinate the transcription of rRNA and translation of ribosome protein.

Ribosomal protein S6 is well-characterized downstream molecule that is regulated by $\mathrm{S} 6 \mathrm{~K}$ and $\mathrm{S} 6 \mathrm{~K}$ is the only proven S6 S240/244 phosphorylating enzyme in mammalian cells. In recent years, research demonstrated new evidence for cell cycle-dependent, rapamycin-resistant phosphorylation of ribosomal protein S6 at S240/244 (Rosner et al., 2010). Furthermore, RSK (90 kDa ribosomal S6 kinase) was found to contribute to rpS6 phosphorylation in response to agonists or oncogenes that activate the MAPK pathway (Romeo et al., 2012). Understanding the RSK- and S6K-specific phosphorylation events on S6 will be necessary to determine the complexities of S6 regulation and function. S6K1-mediated phosphorylation of S6 was believed for quite a while to promote the translation of TOP mRNAs (5'-terminal oligopyrimidine mRNAs) that encode ribosomal proteins and translation factors, but some new evidence indicated that phosphorylation of S6 was not critical for TOP translation (Magnuson et al., 2012). Does the S6K-rpS6 axis promote TOP mRNA translation? It is an intriguing idea that will require further testing. These findings provide important insights into the regulation of S6 and allow a more detailed understanding of function.

In this study, we examined the molecular characterization and expression of S6 in goat, demonstrating that it was expressed in indicated goat tissues. Further study is needed to determine how the S6 works in Inner Mongolia Cashmere goat cells.

\section{CONCLUSION}

In conclusion, we cloned S6 cDNA from Inner Mongolia Cashmere goats. The cloned gene includes an open reading frame of $750 \mathrm{bp}$ and a $58 \mathrm{bp}$ - residue fragment of the 3'untranslated region. The S6 gene was expressed in all the tested tissues and the mRNA yield was greater in heart and kidney compared with brain, liver, spleen, lung and testis.

\section{ACKNOWLEDGEMENTS}

This work was supported by grant from Natural Sciences Foundation of China (No. 31160469), Natural Sciences Foundation of Inner Mongolia, China (No. 2011MS0521), a graduate student research project of Inner Mongolia University, and Major Projects for New Varieties of Genetically Modified Organisms (No.2013ZX08008002).

\section{REFERENCES}

Arnold, K., L. Bordoli, J. Kopp, and T. Schwede. 2006. The SWISS-MODEL workspace: a web-based environment for protein structure homology modelling. Bioinformatics 22:195201.

Bartel, D. P. 2009. MicroRNAs: target recognition and regulatory functions. Cell 136:215-233.

Boylan, J. M., P. Anand, and P. A. Gruppuso. 2001. Ribosomal protein S6 phosphorylation and function during late gestation liver development in the rat. J. Biol. Chem. 276:44457-44463.

Chen, W. and D. P. Dittmer. 2011. Ribosomal protein S6 interacts with the latency-associated nuclear antigen of Kaposi's sarcoma-associated herpesvirus. J. Virol. 85:9495-9505.

Fenton, T. R. and I. T. Gout. 2011. Functions and regulation of the 70kDa ribosomal S6 kinases. Int. J. Biochem. Cell Biol. 43:4759.

Flotow, H. and G. Thomas. 1992. Substrate recognition determinants of the mitogen-activated 70K S6 kinase from rat liver. J. Biol. Chem. 267:3074-3078.

Guex, N. and M. C. Peitsch. 1997. SWISSCMMODEL and the Swiss@ $P$ Pdb Viewer: an environment for comparative protein modeling. Electrophoresis 18:2714-2723.

Hay, N. and N. Sonenberg. 2004. Upstream and downstream of mTOR. Genes Dev. 18:1926-1945.

Hutchinson, M. S., Y. Figenschau, B. Almas, I. Njolstad, and R. Jorde. 2011. Serum 25-hydroxyvitamin D levels in subjects with reduced glucose tolerance and type 2 diabetes - the 
Tromso OGTT-study. Internationale Zeitschrift fur Vitaminund Ernahrungsforschung Journal international de vitaminologie et de nutrition. Int. J. Vit. Nutr. Res. 81:317-327.

Iwenofu, O. H., R. D. Lackman, A. P. Staddon, D. G. Goodwin, H. M. Haupt, and J. S. Brooks. 2008. Phospho-S6 ribosomal protein: a potential new predictive sarcoma marker for targeted mTOR therapy. Modern pathology: An official journal of the United States and Canadian Academy of Pathology, Inc. 21:231-237.

Jefferies, H. B., S. Fumagalli, P. B. Dennis, C. Reinhard, R. B. Pearson, and G. Thomas. 1997. Rapamycin suppresses 5'TOP mRNA translation through inhibition of p70s6k. EMBO J. 16:3693-3704.

Kahvejian, A., G. Roy, and N. Sonenberg. 2001. The mRNA closed-loop model: the function of PABP and PABPinteracting proteins in mRNA translation. Cold Spring Harb. Symp. Quant. Biol. 66:293-300.

Klann, E., M. D. Antion, J. L. Banko, and L. Hou. 2004. Synaptic plasticity and translation initiation. Learn. Mem. 11:365-372.

Krieg, J., J. Hofsteenge, and G. Thomas. 1988. Identification of the $40 \mathrm{~S}$ ribosomal protein S6 phosphorylation sites induced by cycloheximide. J. Biol. Chem. 263:11473-11477.

Kundu-Michalik, S., M. A. Bisotti, E. Lipsius, A. Bauche, A. Kruppa, T. Klokow, G. Kammler, and J. Kruppa. 2008 Nucleolar binding sequences of the ribosomal protein S6e family reside in evolutionary highly conserved peptide clusters. Mol. Biol. Evol. 25:580-590.

Laplante, M. and D. M. Sabatini. 2012. mTOR signaling in growth control and disease. Cell 149:274-293.

Ledda, M., M. Di Croce, B. Bedini, F. Wannenes, M. Corvaro, P. P. Boyl, S. Caldarola, F. Loreni, and F. Amaldi. 2005. Effect of 3'UTR length on the translational regulation of 5'-terminal oligopyrimidine mRNAs. Gene 344:213-220.

Li, Y., S. Mitsuhashi, M. Ikejo, N. Miura, T. Kawamura, T. Hamakubo, and M. Ubukata. 2012. Relationship between ATM and ribosomal protein S6 revealed by the chemical inhibition of Ser/Thr protein phosphatase type 1. Biosci. Biotechnol. Biochem. 76:486-494.

Magnuson, J., F. Leonessa, and G. S. Ling. 2012. Neuropathology of explosive blast traumatic brain injury. Curr. Neurol. Neurosci. Rep. 12:570-579.

Mayer, C., J. Zhao, X. Yuan, and I. Grummt. 2004. mTORdependent activation of the transcription factor TIF-IA links rRNA synthesis to nutrient availability. Genes Dev. 18:423-434.

Mazumder, B., V. Seshadri, and P. L. Fox. 2003. Translational control by the 3'-UTR: the ends specify the means. Trends Biochem. Sci. 28:91-98.

Parkhitko, C. A., C. O. Favorova, and E. P. Henske. 2011. Rabin8 protein interacts with GTPase Rheb and inhibits phosphorylation of Ser235/Ser236 in small ribosomal subunit protein S6. Acta Nat. 3:71-76.
Pende, M., S. H. Um, V. Mieulet, M. Sticker, V. L. Goss, J. Mestan, M. Mueller, S. Fumagalli, S.C. Kozma, and G. Thomas. 2004. S6K1(-/-)/S6K2(-/-) mice exhibit perinatal lethality and rapamycin-sensitive 5'-terminal oligopyrimidine mRNA translation and reveal a mitogen-activated protein kinasedependent S6 kinase pathway. Mol. Cell. Biol. 24:3112-3124.

Peterson, R. T., B. N. Desai, J. S. Hardwick, and S. L. Schreiber. 1999. Protein phosphatase $2 \mathrm{~A}$ interacts with the $70-\mathrm{kDa}$ S6 kinase and is activated by inhibition of FKBP12rapamycinassociated protein. Proc. Natl. Acad. Sci. USA. 96:4438-4442.

Proud, C. G. 2002. Regulation of mammalian translation factors by nutrients. Eur. J. Biochem. 269:5338-5349.

Romeo, S., C. Maglio, M. A. Burza, C. Pirazzi, K. Sjoholm, P. Jacobson, P. A. Svensson, M. Peltonen, L. Sjostrom, and L. M. Carlsson. 2012. Cardiovascular events after bariatric surgery in obese subjects with type 2 diabetes. Diabetes Care. 35:26132617.

Rosner, M., C. Fuchs, H. Dolznig, and M. Hengstschlager. 2011. Different cytoplasmic/nuclear distribution of S6 protein phosphorylated at S240/244 and S235/236. Amino Acids 40:595-600.

Rosner, S., J. Konnerth, B. Plank, D. Salaberger, and C. Hansmann. 2010. Radial shrinkage and ultrasound acoustic emissions of fresh versus pre-dried Norway spruce sapwood. Trees (Berl West). 24:931-940.

Ruvinsky, I., M. Katz, A. Dreazen, Y. Gielchinsky, A. Saada, N. Freedman, E. Mishani, G. Zimmerman, J. Kasir, and O. Meyuhas. 2009. Mice deficient in ribosomal protein S6 phosphorylation suffer from muscle weakness that reflects a growth defect and energy deficit. PLoS One. 4:e5618.

Ruvinsky, I. and O. Meyuhas. 2006. Ribosomal protein S6 phosphorylation: from protein synthesis to cell size. Trends Biochem. Sci. 31:342-348.

Schmelzle, T. and M. N. Hall. 2000. TOR, a central controller of cell growth. Cell 103:253-262.

Schwede, T., J. Kopp, N. Guex, and M.C. Peitsch. 2003. SWISSMODEL: an automated protein homology-modeling server. Nucl. Acids Res. 31:3381-3385.

Stark, A., J. Brennecke, N. Bushati, R. B. Russell, and S. M. Cohen. 2005. Animal MicroRNAs confer robustness to gene expression and have a significant impact on 3'UTR evolution. Cell 123:1133-1146.

Svitkin, Y. V. and N. Sonenberg. 2004. An efficient system for capand poly(A)-dependent translation in vitro. Methods Mol. Biol. 257:155-170.

Tanguay, R. L. and D. R. Gallie. 1996. Translational efficiency is regulated by the length of the 3 ' untranslated region. Mol. Cell. Biol. 16:146-156.

Wullschleger, S., R. Loewith, and M. N. Hall. 2006. TOR signaling in growth and metabolism. Cell 124:471-484.

Zhang, R. and B. Su. 2009. Small but influential: the role of microRNAs on gene regulatory network and 3'UTR evolution. J. Genet. Genomics 36:1-6. 